PROCEEDINGS OF THE

AMERICAN MATHEMATICAL SOCIETY

Volume 128, Number 5, Pages 1361-1369

S 0002-9939(99)05156-4

Article electronically published on August 5, 1999

\title{
ON THE STABILITY \\ OF APPROXIMATELY ADDITIVE MAPPINGS
}

\author{
YANG-HI LEE AND KIL-WOUNG JUN \\ (Communicated by Dale Alspach)
}

\begin{abstract}
In this paper we prove a generalization of the stability of approximately additive mappings in the spirit of Hyers, Ulam and Rassias.
\end{abstract}

\section{INTRODUCTION}

In 1941 Hyers [3] showed that if $\delta>0$ and $f: E_{1} \rightarrow E_{2}$, with $E_{1}$ and $E_{2}$ Banach spaces, such that

$$
\|f(x+y)-f(x)-f(y)\| \leq \delta, \quad \text { for all } \quad x, y \in E_{1},
$$

then there exists a unique additive mapping $T: E_{1} \rightarrow E_{2}$ such that

$$
\|f(x)-T(x)\| \leq \delta,
$$

for all $x \in E_{1}$, and if $f(t x)$ is continuous in $t$ for each fixed $x$, then $T$ is a linear mapping.

Rassias [6] and Gajda [1] gave some generalizations of the Hyers' result in the following ways : Let $f: E_{1} \rightarrow E_{2}$ be a mapping such that $f(t x)$ is continuous in $t$ for each fixed $x$. Assume that there exist $\theta \geq 0$ and $p \neq 1$ such that

$$
\frac{\|f(x+y)-f(x)-f(y)\|}{\|x\|^{p}+\|y\|^{p}} \leq \theta, \quad \text { for all } x, y \in E_{1} .
$$

Then there exists a unique linear mapping $T: E_{1} \rightarrow E_{2}$ such that

$$
\frac{\|T(x)-f(x)\|}{\|x\|^{p}} \leq \frac{2 \theta}{2-2^{p}}, \quad \text { for all } x \in E_{1} .
$$

However, it was showed that the similar result for the case $p=1$ does not hold (see [7]). Recently, Găvruta [2] also obtained a further generalization of the HyersRassias theorem : Let $G$ be an abelian group and $X$ a Banach space. Denote by $\varphi: G \times G \rightarrow[0, \infty)$ a mapping such that

$$
\tilde{\varphi}(x, y)=\sum_{k=0}^{\infty} 2^{-k} \varphi\left(2^{k} x, 2^{k} y\right)<\infty
$$

for all $x, y \in G$. Suppose $f: G \rightarrow X$ is a mapping satisfying

$$
\|f(x+y)-f(x)-f(y)\| \leq \varphi(x, y)
$$

Received by the editors February 25, 1998 and, in revised form, June 22, 1998.

1991 Mathematics Subject Classification. Primary 47H15.

(C)2000 American Mathematical Society 
for all $x, y \in G$. Then there exists a unique additive mapping $T: G \rightarrow X$ such that

$$
\|f(x)-T(x)\| \leq \frac{1}{2} \tilde{\varphi}(x, x) \quad \text { for all } x \in G .
$$

In this paper we generalize the results of Hyers, Rassias and Găvruta.

\section{MAin RESUlts}

Throughout this paper, let $a$ be a fixed rational number with $a>1$. If $a$ is not an integer, there exist unique nonnegative integers $b, p$ and $q$ such that $a=b+q / p$, $0<q / p<1$ and $(p, q)=1$. If $a$ is an integer, we let $a=b$. We denote by $G$ a vector space, by $X$ a Banach space, and by $\varphi: G \times G \rightarrow[0, \infty)$ a mapping such that

$$
\tilde{\varphi}(x, y)=\sum_{k=0}^{\infty} a^{-k} \varphi\left(a^{k} x, a^{k} y\right)<\infty
$$

for all $x, y \in G$. In particular, when $a=2$, we denote $\tilde{\varphi}(x, y)$ by $\tilde{\varphi}_{2}(x, y)$. We also assume that $\sum_{i=2}^{n}(\cdot)=0$ if $n<2$.

Theorem 2.1. Let $f: G \rightarrow X$ be such that

$$
\|f(x+y)-f(x)-f(y)\| \leq \varphi(x, y), \text { for all } x, y \in G .
$$

Then there exists a unique additive mapping $T: G \rightarrow X$ such that

$$
\begin{aligned}
\|T(x)-f(x)\| & \leq a^{-1} \tilde{\varphi}\left(\frac{q}{p} x, b x\right)+a^{-1} \frac{q}{p} \sum_{i=2}^{p} \tilde{\varphi}\left(\frac{1}{p} x, \frac{i-1}{p} x\right) \\
& +a^{-1} \sum_{i=2}^{q} \tilde{\varphi}\left(\frac{1}{p} x, \frac{i-1}{p} x\right)+a^{-1} \sum_{i=2}^{b} \tilde{\varphi}(x,(i-1) x),
\end{aligned}
$$

for all $x \in G$.

Proof. We first prove the case that $a$ is not an integer. Putting $y=i x$ in (2), we have

$$
\|f((i+1) x)-f(x)-f(i x)\| \leq \varphi(x, i x), \quad \text { for all } x \in G, i \in N .
$$

Thus

$$
\begin{aligned}
\|f((k+1) x)-(k+1) f(x)\| & \leq \sum_{i=1}^{k}\|f((i+1) x)-f(x)-f(i x)\| \\
& \leq \sum_{i=2}^{k+1} \varphi(x,(i-1) x)
\end{aligned}
$$

for all $x \in G, k \in N$. From (4) it follows that

$$
\left\|a^{-1} f(b x)-a^{-1} b f(x)\right\| \leq \sum_{i=2}^{b} a^{-1} \varphi(x,(i-1) x) .
$$

Replacing $x$ by $\frac{q}{p} x$ and $y$ by $b x,(2)$ gives

$$
\left\|a^{-1} f(a x)-a^{-1} f\left(\frac{q}{p} x\right)-a^{-1} f(b x)\right\| \leq a^{-1} \varphi\left(\frac{q}{p} x, b x\right) .
$$


Replacing $x$ by $\frac{1}{p} x$ and $k+1$ by $p,(4)$ gives

$$
\left\|f(x)-p f\left(\frac{1}{p} x\right)\right\| \leq \sum_{i=2}^{p} \varphi\left(\frac{1}{p} x, \frac{i-1}{p} x\right) .
$$

Replacing $x$ by $\frac{1}{p} x$ and $k+1$ by $q$, (4) gives

$$
\left\|f\left(\frac{q}{p} x\right)-q f\left(\frac{1}{p} x\right)\right\| \leq \sum_{i=2}^{q} \varphi\left(\frac{1}{p} x, \frac{i-1}{p} x\right) .
$$

From (7) and (8), we obtain

$$
\begin{aligned}
a^{-1}\left\|\frac{q}{p} f(x)-f\left(\frac{q}{p} x\right)\right\| & \leq a^{-1} \frac{q}{p} \sum_{i=2}^{p} \varphi\left(\frac{1}{p} x, \frac{i-1}{p} x\right) \\
& +a^{-1} \sum_{i=2}^{q} \varphi\left(\frac{1}{p} x, \frac{i-1}{p} x\right) .
\end{aligned}
$$

From (5), (6) and (9), we get

$$
\begin{aligned}
\left\|a^{-1} f(a x)-f(x)\right\| & \leq a^{-1}\left\|f(a x)-f\left(\frac{q}{p} x\right)-f(b x)\right\| \\
& +a^{-1}\left\|\frac{q}{p}(x)-f\left(\frac{q}{p} x\right)\right\|+a^{-1}\|f(b x)-b f(x)\| \\
& \leq a^{-1}\left[\varphi\left(\frac{q}{p} x, b x\right)+\frac{q}{p} \sum_{i=2}^{p} \varphi\left(\frac{1}{p} x, \frac{i-1}{p} x\right)\right. \\
& \left.+\sum_{i=2}^{q} \varphi\left(\frac{1}{p} x, \frac{i-1}{p} x\right)+\sum_{i=2}^{b} \varphi(x,(i-1) x)\right] .
\end{aligned}
$$

Replacing $x$ by $a^{k-1} x,(10)$ gives

$$
\begin{aligned}
& \left\|a^{-1} f\left(a^{k} x\right)-f\left(a^{k-1} x\right)\right\| \\
& \leq a^{-1}\left[\varphi\left(a^{k-1} \frac{q}{p} x, a^{k-1} b x\right)+\frac{q}{p} \sum_{i=2}^{p} \varphi\left(a^{k-1} \frac{1}{p} x, a^{k-1} \frac{i-1}{p} x\right)\right. \\
& \left.+\sum_{i=2}^{q} \varphi\left(a^{k-1} \frac{1}{p} x, a^{k-1} \frac{i-1}{p} x\right)+\sum_{i=2}^{b} \varphi\left(a^{k-1} x, a^{k-1}(i-1) x\right)\right] .
\end{aligned}
$$


From (11) we obtain

$$
\begin{aligned}
\left\|a^{-n} f\left(a^{n} x\right)-f(x)\right\| & \leq \sum_{k=1}^{n} a^{-k+1}\left\|a^{-1} f\left(a^{k} x\right)-f\left(a^{k-1} x\right)\right\| \\
& \leq \sum_{k=1}^{n} a^{-k} \varphi\left(a^{k-1} \frac{q}{p} x, a^{k-1} b x\right) \\
& +\frac{q}{p} \sum_{i=2}^{p} \sum_{k=1}^{n} a^{-k} \varphi\left(a^{k-1} \frac{1}{p} x, a^{k-1} \frac{i-1}{p} x\right) \\
& +\sum_{i=2}^{q} \sum_{k=1}^{n} a^{-k} \varphi\left(a^{k-1} \frac{1}{p} x, a^{k-1} \frac{i-1}{p} x\right) \\
& +\sum_{i=2}^{b} \sum_{k=1}^{n} a^{-k} \varphi\left(a^{k-1} x, a^{k-1}(i-1) x\right)
\end{aligned}
$$

for all $x \in G$.

We claim that the sequence $\left\{a^{-n} f\left(a^{n} x\right)\right\}$ is a Cauchy sequence. Indeed, for $n>m$, we have

$$
\begin{aligned}
\left\|a^{-n} f\left(a^{n} x\right)-a^{-m} f\left(a^{m} x\right)\right\| & \leq \sum_{k=m+1}^{n} a^{-k+1}\left\|a^{-1} f\left(a^{k} x\right)-f\left(a^{k-1} x\right)\right\| \\
& \leq \sum_{k=m+1}^{n} a^{-k} \varphi\left(a^{k-1} \frac{q}{p} x, a^{k-1} b x\right) \\
& +\frac{q}{p} \sum_{i=2}^{p} \sum_{k=m+1}^{n} a^{-k} \varphi\left(a^{k-1} \frac{1}{p} x, a^{k-1} \frac{i-1}{p} x\right) \\
& +\sum_{i=2}^{q} \sum_{k=m+1}^{n} a^{-k} \varphi\left(a^{k-1} \frac{1}{p} x, a^{k-1} \frac{i-1}{p} x\right) \\
& +\sum_{i=2}^{b} \sum_{k=m+1}^{n} a^{-k} \varphi\left(a^{k-1} x, a^{k-1}(i-1) x\right)
\end{aligned}
$$

for all $x \in G$. Taking the limit in (13) as $m \rightarrow \infty$ we obtain

$$
\lim _{m \rightarrow \infty}\left\|a^{-n} f\left(a^{n} x\right)-a^{-m} f\left(a^{m} x\right)\right\|=0 .
$$

Since $X$ is a Banach space, the sequence $\left\{a^{-n} f\left(a^{n} x\right)\right\}$ converges for every $x \in G$. Denote

$$
T(x)=\lim _{n \rightarrow \infty} \frac{f\left(a^{n} x\right)}{a^{n}} .
$$

From (2) we have

$$
\begin{aligned}
\| a^{-n} f\left(a^{n} x+a^{n} y\right)-a^{-n} f\left(a^{n} x\right) & -a^{-n} f\left(a^{n} y\right) \| \\
& \leq a^{-n} \varphi\left(a^{n} x, a^{n} y\right) \quad \text { for all } x, y \in G .
\end{aligned}
$$

From (1) it follows that

$$
\lim _{n \rightarrow \infty} a^{-n} \varphi\left(a^{n} x, a^{n} y\right)=0 .
$$


Then (14) implies

$$
\|T(x+y)-T(x)-T(y)\|=0 .
$$

To prove (3), taking the limit in (12) as $n \rightarrow \infty$, we obtain

$$
\begin{aligned}
\|T(x)-f(x)\| & \leq a^{-1} \tilde{\varphi}\left(\frac{q}{p} x, b x\right)+a^{-1} \frac{q}{p} \sum_{i=2}^{p} \tilde{\varphi}\left(\frac{1}{p} x, \frac{i-1}{p} x\right) \\
& +a^{-1} \sum_{i=2}^{q} \tilde{\varphi}\left(\frac{1}{p} x, \frac{i-1}{p} x\right)+a^{-1} \sum_{i=2}^{b} \tilde{\varphi}(x,(i-1) x) \quad \text { for all } x \in G .
\end{aligned}
$$

It remains to show that $T$ is uniquely defined. Let $F: G \rightarrow X$ be another additive mapping satisfying (3). Then

$$
\begin{aligned}
& \|T(x)-F(x)\|=\left\|a^{-n} T\left(a^{n} x\right)-a^{-n} F\left(a^{n} x\right)\right\| \\
& \leq\left\|a^{-n} T\left(a^{n} x\right)-a^{-n} f\left(a^{n} x\right)\right\|+\left\|a^{-n} f\left(a^{n} x\right)-a^{-n} F\left(a^{n} x\right)\right\| \\
& \leq 2\left[a^{-n-1} \tilde{\varphi}\left(a^{n} \frac{q}{p} x, a^{n} b x\right)+a^{-n-1} \frac{q}{p} \sum_{i=2}^{p} \tilde{\varphi}\left(a^{n} \frac{1}{p} x, a^{n} \frac{i-1}{p} x\right)\right. \\
& \left.+a^{-n-1} \sum_{i=2}^{q} \tilde{\varphi}\left(a^{n} \frac{1}{p} x, a^{n} \frac{i-1}{p} x\right)+a^{-n-1} \sum_{i=2}^{b} \tilde{\varphi}\left(a^{n} x, a^{n}(i-1) x\right)\right] \\
& =2 a^{-1}\left[\sum_{j=n}^{\infty} a^{-j} \varphi\left(a^{j} \frac{q}{p} x, a^{j} b x\right)+\frac{q}{p} \sum_{i=2}^{p} \sum_{j=n}^{\infty} a^{-j} \varphi\left(a^{j} \frac{1}{p} x, a^{j} \frac{i-1}{p} x\right)\right. \\
& \left.+\sum_{i=2}^{q} \sum_{j=n}^{\infty} a^{-j} \varphi\left(a^{j} \frac{1}{p} x, a^{j} \frac{i-1}{p} x\right)+\sum_{i=2}^{b} \sum_{j=n}^{\infty} a^{-j} \varphi\left(a^{j} x, a^{j}(i-1) x\right)\right] .
\end{aligned}
$$

Thus

$$
\begin{aligned}
& \|T(x)-F(x)\|=\left\|a^{-n} T\left(a^{n} x\right)-a^{-n} F\left(a^{n} x\right)\right\| \\
& \leq 2 a^{-1}\left[\sum_{j=n}^{\infty} a^{-j} \varphi\left(a^{j} \frac{q}{p} x, a^{j} b x\right)+\frac{q}{p} \sum_{i=2}^{p} \sum_{j=n}^{\infty} a^{-j} \varphi\left(a^{j} \frac{1}{p} x, a^{j} \frac{i-1}{p} x\right)\right. \\
& \left.+\sum_{i=2}^{q} \sum_{j=n}^{\infty} a^{-j} \varphi\left(a^{j} \frac{1}{p} x, a^{j} \frac{i-1}{p} x\right)+\sum_{i=2}^{b} \sum_{j=n}^{\infty} a^{-j} \varphi\left(a^{j} x, a^{j}(i-1) x\right)\right]
\end{aligned}
$$

for all $x \in G$. Taking the limit (15) as $n \rightarrow \infty$ we obtain

$$
T(x)=F(x) \text { for all } x \in G .
$$

Now we prove the case: $a=b$. From (5) we obtain

$$
\left\|a^{-1} f(a x)-f(x)\right\| \leq \sum_{i=2}^{a} a^{-1} \varphi(x,(i-1) x) .
$$

Hence we have

$$
\left\|a^{-n} f\left(a^{n} x\right)-f(x)\right\| \leq \sum_{i=2}^{a} \sum_{k=1}^{n} a^{-k} \varphi\left(a^{k-1} x, a^{k-1}(i-1) x\right)
$$


for all $x \in G$. Denote

$$
T(x)=\lim _{n \rightarrow \infty} \frac{f\left(a^{n} x\right)}{a^{n}} .
$$

Taking the limit in $\left(12^{\prime}\right)$ as $n \rightarrow \infty$, we obtain

$$
\|T(x)-f(x)\| \leq a^{-1} \sum_{i=2}^{a} \tilde{\varphi}(x,(i-1) x) \quad \text { for all } x \in G .
$$

It is easy to show that $T$ is uniquely defined.

Lemma 2.2. Let $T: G \rightarrow X$ be an additive mapping and let $x_{0} \in G$. If there are an interval $(c, d)$ and $y \in G$ such that $C=\left\{\left\|T\left(u x_{0}+y\right)\right\|: u \in(c, d)\right\}$ is bounded, then

$$
T\left(u x_{0}\right)=u T\left(x_{0}\right) \quad \text { for all real numbers } u .
$$

Proof. Assume that there exists a real number $r$ such that $T\left(r x_{0}\right) \neq r T\left(x_{0}\right)$. Let $m=\left\|T\left(r x_{0}\right)-r T\left(x_{0}\right)\right\|$. Let $\left\{r_{n}\right\}$ be a rational number sequence such that

$$
\left\|\left(r-r_{n}\right) T\left(x_{0}\right)\right\| \leq m / 2 \text { and } \lim _{n \rightarrow \infty} r_{n}=r .
$$

Choose a rational number sequence $\left\{r_{n}^{\prime}\right\}$ such that $r_{n}^{\prime}\left(r-r_{n}\right) \in(c, d)$ and $\lim _{n \rightarrow \infty} r_{n}^{\prime}$ $=\infty$. Since

$$
\begin{aligned}
& \left\|T\left(r_{n}^{\prime}\left(r-r_{n}\right) x_{0}+y\right)-r_{n}^{\prime} r T\left(x_{0}\right)+r_{n}^{\prime} r_{n} T\left(x_{0}\right)-T(y)\right\| \\
& \quad=\left\|r_{n}^{\prime} T\left(r x_{0}\right)-r_{n}^{\prime} r T\left(x_{0}\right)\right\| \\
& \quad=\left|r_{n}^{\prime}\right| m,
\end{aligned}
$$

we have

$$
\left\|T\left(r_{n}^{\prime}\left(r-r_{n}\right) x_{0}+y\right)\right\| \geq\left|r_{n}^{\prime}\right|(m / 2)-\|T(y)\| \quad \text { for all } n \in N .
$$

This contradicts the fact that $C$ is bounded.

Remarks. In Theorem 2.1, (a) if there exist an interval $(c, d)$ and $\varepsilon>0$ such that $\left\{\left\|f\left(u x_{0}\right)\right\|: u \in(c, d)\right\}$ and $\left\{\tilde{\varphi}\left(s x_{0}, t x_{0}\right): d /(p+\varepsilon) \leq s, t \leq(b-1) d\right\}$ are bounded for a fixed $x_{0}$, then $T\left(r x_{0}\right)=r T\left(x_{0}\right)$ for all real numbers $r$. In fact, choose an interval $\left(c^{\prime}, d^{\prime}\right) \subset(c, d) \cap(d p /(p+\varepsilon), d)$. From (3) we obtain $C=\left\{\left\|T\left(u x_{0}\right)\right\|: u \in\left(c^{\prime}, d^{\prime}\right)\right\}$ is bounded.

(b) If $G$ is a normed space and $f(t x)$ is continuous in $t$ for each fixed $x$ and $\tilde{\varphi}$ is bounded on $G \times G$, then $T$ is linear by (a).

Theorem 2.3. Let $G$ be a normed space and $f$ be as in Theorem 2.1. If $f$ is bounded for some open subset $A$ of $G$ and $\tilde{\varphi}$ is bounded on $G \times G$, then there exists a unique continuous linear mapping $T: G \rightarrow X$ such that

$$
\begin{aligned}
\|T(x)-f(x)\| & \leq a^{-1} \tilde{\varphi}\left(\frac{q}{p} x, b x\right)+a^{-1} \frac{q}{p} \sum_{i=2}^{p} \tilde{\varphi}\left(\frac{1}{p} x, \frac{i-1}{p} x\right) \\
& +a^{-1} \sum_{i=2}^{q} \tilde{\varphi}\left(\frac{1}{p} x, \frac{i-1}{p} x\right)+a^{-1} \sum_{i=2}^{b} \tilde{\varphi}(x,(i-1) x) \quad \text { for all } x \in G .
\end{aligned}
$$

Proof. Let $T$ be a mapping as in Theorem 2.1. From (4) we obtain that $T$ is bounded on $A$. Let $z$ be an interior point of $A$. For each fixed $x \in G$ there exists an interval $(c, d)$ such that $\{u x+z: u \in(c, d)\} \subset A$. By the preceding remark, $T$ is linear. Since $T$ is bounded on open set $A, T$ is continuous. 
Corollary 2.4. Let $G$ and $f$ be as in Theorem 2.3. If $f$ is bounded for some open subset $A$ of $G$ and $\tilde{\varphi}_{2}$ is bounded on $A \times A$, then there exists a unique continuous linear mapping $T: G \rightarrow X$ such that

$$
\|T(x)-f(x)\| \leq 2^{-1} \tilde{\varphi}_{2}(x, x) \quad \text { for all } x \in G .
$$

Proof. By Theorem 2.1, there exists a unique additive mapping $T: G \rightarrow X$ such that $\|T(x)-f(x)\| \leq 2^{-1} \tilde{\varphi}_{2}(x, x)$ for all $x \in A$. We can apply the similar method as in Theorem 2.3 .

Theorem 2.5. Let $f: E_{1} \rightarrow E_{2}$ be a mapping with $E_{1}$ and $E_{2}$ Banach spaces. If for each fixed $x, y \in E_{1}$ there exist real numbers $\theta_{x y}, p_{x y}, s_{x y}$ such that $0 \leq p_{x y}<1$ and

$$
\|f(t x+t y)-f(t x)-f(t y)\| \leq \theta_{x y}\left(\|t x\|^{p_{x y}}+\|t y\|^{p_{x y}}\right) \quad \text { for } t>s_{x y},
$$

then there exists a unique additive mapping $T: E_{1} \rightarrow E_{2}$ such that

$$
\|T(t x)-f(t x)\| \leq \frac{2 \theta_{x x}\|t x\|^{p_{x x}}}{2-2^{p_{x x}}} \quad \text { for } t>s_{x x}
$$

for all $x \in E_{1}$. In particular, if for a fixed $x_{0} \in E_{1}$ there exist real numbers $M_{x_{0}}, s_{x_{0}}$ such that $\left\|f\left(t x_{0}\right)\right\| / t<M_{x_{0}}$ for $t>s_{x_{0}}$, then $T\left(r x_{0}\right)=r T\left(x_{0}\right)$ for all real numbers $r$.

Proof. Let

$$
\varphi(t x, t y)=\|f(t x+t y)-f(t x)-f(t y)\| .
$$

Then $\tilde{\varphi}_{2}(x, y)<\infty$ for all $x, y \in E_{1}$ and $2^{-1} \tilde{\varphi}_{2}(t x, t x)<2 \theta_{x x}\|t x\|^{p_{x x}} /\left(2-2^{p_{x x}}\right)$ for $t>s_{x x}$ for each $x \in E_{1}$. By Theorem 2.1 there exists a unique additive mapping $T: E_{1} \rightarrow E_{2}$ such that

$$
\|T(t x)-f(t x)\| \leq \frac{2 \theta_{x x}\|t x\|^{p_{x x}}}{2-2^{p_{x x}}} \quad \text { for } t>s_{x x}
$$

for all $x, y \in E_{1}$. If for a fixed $x_{0} \in E_{1}$ there exist real numbers $M_{x_{0}}, s_{x_{0}}$ with $\left\|f\left(t x_{0}\right)\right\| / t<M_{x_{0}}$ for $t>s_{x_{0}}$, then

$$
\left\|T\left(t x_{0}\right)\right\| \leq \frac{2 \theta_{x_{0} x_{0}}\left\|t x_{0}\right\|^{p_{x_{0} x_{0}}}}{2-2^{p_{x_{0} x_{0}}}}+M_{x_{0}} t \text { for } t>\max \left(s_{x_{0} x_{0}}, s_{x_{0}}\right) .
$$

Therefore $\left\{\left\|T\left(u x_{0}\right)\right\|: u \in\left(\max \left(s_{x_{0} x_{0}}, s_{x_{0}}\right), 2 \max \left(s_{x_{0} x_{0}}, s_{x_{0}}\right)\right)\right\}$ is bounded. Apply Lemma 2.2 .

The following theorem is a generalization of Theorem 1 in [4].

Theorem 2.6. Let a function $\psi: R^{+} \rightarrow R^{+}$satisfy

(i) $\psi(t s) \leq \psi(t) \psi(s)$ for all $t, s \in R^{+}$and

(ii) $\lim _{t \rightarrow \infty} \psi(t) / t=0$

and let $f: E_{1} \rightarrow E_{2}$ be a mapping with $E_{1}$ and $E_{2}$ Banach spaces. If for each fixed $x, y \in E_{1}$, there exists a real number $\theta_{x y}$ such that

$$
\|f(t x+t y)-f(t x)-f(t y)\| \leq \theta_{x y}(\psi(\|t x\|)+\psi(\|t y\|)) \text { for all } t \in R^{+},
$$


then there exist a unique additive mapping $T: E_{1} \rightarrow E_{2}$ and a rational number $a>1$ such that

$$
\begin{aligned}
\|f(t x)-T(t x)\| & \leq a^{-1}\left(1-\frac{\psi(a)}{a}\right)\left[\theta_{(q / p) x, b x}\left(\psi\left(\left\|\frac{q}{p} t x\right\|\right)+\psi(\|b t x\|)\right)\right. \\
& +\frac{q}{p} \sum_{i=2}^{p} \theta_{(1 / p) x,(i-1) x / p}\left(\psi\left(\left\|\frac{1}{p} t x\right\|\right)+\psi\left(\left\|\frac{i-1}{p} t x\right\|\right)\right) \\
& +\sum_{i=2}^{q} \theta_{(1 / p) x,(i-1) x / p}\left(\psi\left(\left\|\frac{1}{p} t x\right\|\right)+\psi\left(\left\|\frac{i-1}{p} t x\right\|\right)\right) \\
& \left.+\sum_{i=2}^{b} \theta_{x,(i-1) x}(\psi(\|t x\|)+\psi(\|(i-1) t x\|))\right] .
\end{aligned}
$$

In particular, if for each fixed $x \in E_{1}$ there exist positive real numbers $c_{x}, d_{x}$ such that $A_{x}=\left\{\|f(u x)\|: u \in\left(c_{x}, d_{x}\right)\right\}$ is bounded, then $T$ is linear.

Proof. From (ii), there exists a rational number $a$ such that $\psi(a)<a$. Let $\varphi(x, y)=$ $\|f(t x+t y)-f(t x)-f(t y)\|$. From (i) we get

$$
\begin{aligned}
\tilde{\varphi}(t x, t y) & =\sum_{n=1}^{\infty} a^{-n} \varphi\left(a^{n} t x, a^{n} t y\right) \\
& \leq \sum_{n=1}^{\infty} a^{-n} \theta_{x y}\left(\psi\left(\left\|a^{n} t x\right\|\right)+\psi\left(\left\|a^{n} t y\right\|\right)\right) \\
& \leq \sum_{n=1}^{\infty}(\psi(a) / a)^{n} \theta_{x y}(\psi(\|t x\|)+\psi(\|t y\|)) \\
& =\frac{\theta_{x y}(\psi(\|t x\|)+\psi(\|t y\|))}{1-\psi(a) / a}<\infty
\end{aligned}
$$

for all $x, y \in E_{1}$ and $t \in R^{+}$. By Theorem 2.1 there exists a unique additive mapping $T: E_{1} \rightarrow E_{2}$ such that

$$
\begin{aligned}
\|f(t x)-T(t x)\| & \leq a^{-1}\left(1-\frac{\psi(a)}{a}\right)\left[\theta_{(q / p) x, b x}\left(\psi\left(\left\|\frac{q}{p} t x\right\|\right)+\psi(\|b t x\|)\right)\right. \\
& +\frac{q}{p} \sum_{i=2}^{p} \theta_{(1 / p) x,(i-1) x / p}\left(\psi\left(\left\|\frac{1}{p} t x\right\|\right)+\psi\left(\left\|\frac{i-1}{p} t x\right\|\right)\right) \\
& +\sum_{i=2}^{q} \theta_{(1 / p) x,(i-1) x / p}\left(\psi\left(\left\|\frac{1}{p} t x\right\|\right)+\psi\left(\left\|\frac{i-1}{p} t x\right\|\right)\right) \\
& \left.+\sum_{i=2}^{b} \theta_{x,(i-1) x}(\psi(\|t x\|)+\psi(\|(i-1) t x\|))\right]
\end{aligned}
$$

for $x \in E_{1}$ and $t \in R^{+}$. Since $\lim _{t \rightarrow \infty} \psi(t) / t=0$, there exists a positive number $M$ such that $\psi(t) / t<1$ for all $t>M$. Choose $N$ such that $c_{x} N>M$. From (17) 
we have

$$
\begin{aligned}
\|f(t x)-T(t x)\| & \leq a^{-1} \psi(t N)\left(1-\frac{\psi(a)}{a}\right)\left[\theta_{(q / p) x, b x}\left(\psi\left(\left\|\frac{q}{p N} x\right\|\right)+\psi\left(\left\|\frac{b}{N} x\right\|\right)\right)\right. \\
& +\frac{q}{p} \sum_{i=2}^{p} \theta_{(1 / p) x,(i-1) x / p}\left(\psi\left(\left\|\frac{1}{p N} x\right\|\right)+\psi\left(\left\|\frac{i-1}{p N} x\right\|\right)\right) \\
& \left.+\sum_{i=2}^{q} \theta_{(1 / p) x,(i-1) x / p}\left(\psi\left(\left\|\frac{1}{p N} x\right\|\right)+\psi\left(\| \frac{i-1}{p N}\right) x \|\right)\right) \\
& \left.\left.+\sum_{i=2}^{b} \theta_{x,(i-1) x}\left(\psi\left(\left\|\frac{1}{N} x\right\|\right)+\psi\left(\| \frac{i-1}{N}\right) x \|\right)\right)\right] .
\end{aligned}
$$

Since $\psi(N t)<N t$ for all $t \in\left(c_{x}, d_{x}\right)$, the right-hand side of the inequality of (18) is bounded for $t \in\left(c_{x}, d_{x}\right)$. From $A_{x}=\left\{\|f(u x)\|: u \in\left(c_{x}, d_{x}\right)\right\}$ is bounded, $C_{x}=\left\{\|T(u x)\|: u \in\left(c_{x}, d_{x}\right)\right\}$ is bounded. Applying Lemma 2.2, $T$ is linear.

\section{REFERENCES}

[1] Z. Gajda, On stability of additive mappings, Internat. J. Math. Sci 14 (1991), 431-434. MR 92e:39029

[2] P. Găvruta, A generalization of the Hyers-Ulam-Rassias stability of approximately additive mappings, J. of Math. Anal. and Appl. 184 (1994), 431-436. MR 95e:47089

[3] D. H. Hyers, On the stability of the linear functional equation, Proc. Natl. Acad. Sci. U.S.A. 27 (1941), 222-224. MR 2:315a

[4] G. Isac and Th. M. Rassias, On the Hyers-Ulam stability of $\psi$-additive mappings, J. Approx. Theory 72 (1993), 131-137. [MR 94b:39043

[5] G. Isac and Th. M. Rassias, Functional inequalities for approximately additive mappings, In: Stability of Mappings of Hyers-Ulam Type (Th. M. Rassias and J. Tabor, eds.), Hadronic Press, Palm Harbor, Fl. (1994), 117-125. MR 95j:39048

[6] Th. M. Rassias, On the stability of the linear mapping in Banach spaces, Proc. Amer. Math. Soc. 72 (1978), 297-300. MR 80d:47094

[7] Th. M. Rassias and P. Šemrl, On the behavior of mappings which does not satisfy Hyers-Ulam stability, Proc. Amer. Math. Soc. 114 (1992), 989-993. MR 92g:47101

[8] S. M. Ulam, "Problems in Modern Mathematics", Chap. VI, Science eds., Wiley, Newyork, 1960. MR 43:6031

Department of Mathematics Education, Konguu National University of Education, Kongue 314-060, Republic of Korea

E-mail address: lyhmzi@kongjuw2.kongju-e.ac.kr

Department of Mathematics, Chungnam National University, Taejon 305-764, RepubLIC OF KOREA

E-mail address: kwjun@math.chungnam.ac.kr 